\title{
Sharp Bounds for the Oriented Diameters of Interval Graphs and 2-Connected Proper Interval Graphs
}

\author{
Jing Huang ${ }^{1}$ and Dong $\mathrm{Ye}^{2}$ \\ ${ }^{1}$ Department of Mathematics and Statistics, University of Victoria, \\ P.O. Box 3045, Victoria, B.C., Canada, V8W 3P4 \\ jing@math.uvic.ca \\ 2 School of mathematics and Statistics, Lanzhou University, \\ Lanzhou, Gansu, 730000, People's Republic of China \\ dye@lzu.edu.cn
}

\begin{abstract}
The diameter $\operatorname{diam}(H)$ of a (directed) graph $H$ is the maximum value computed over the lengths of all shortest (directed) paths in $H$. Given a bridgeless connected graph $G$, the oriented diameter $O D(G)$ is given by $O D(G)=\min \{\operatorname{diam}(H): H$ is an orientation of $G\}$. In this paper, we show that $O D(G) \leq\left\lceil\frac{3}{2} \operatorname{diam}(G)\right\rceil+1$ for every connected bridgeless interval graph $G$. Further, for every 2-connected proper interval graph $G$, we show $O D(G) \leq\left\lceil\frac{5}{4} \operatorname{diam}(G)\right\rceil+1$ if $\operatorname{diam}(G) \leq 3$ and $O D(G) \leq\left\lceil\frac{5}{4} \operatorname{diam}(G)\right\rceil$, otherwise. All the bounds are sharp and improve earlier estimations obtained by Fomin et al.
\end{abstract}

Keywords: Diameter, orientation, oriented diameter, interval graph, proper interval graph.

\section{Introduction}

Given a (directed) graph $H$, the distance from a vertex $x$ to a vertex $y$ in $H$, denoted by $d_{H}(x, y)$, is the length of a shortest (directed) path from $x$ to $y$ in $H$. The maximum value of distances, denoted by $\operatorname{diam}(H)$, is called the diameter of $H$. When $H$ is a graph, $\operatorname{diam}(H)$ is finite if and only if $H$ is connected; when $H$ is a directed graph, $\operatorname{diam}(H)$ is finite if and only if $H$ is strongly connected.

Let $G$ be a graph. An orientation of $G$ is a directed graph obtained from $G$ by assigning a direction to each edge of $G$. The oriented diameter of $G, O D(G)$, is given by

$$
O D(G)=\min \{\operatorname{diam}(H): H \text { is an orientation of } G\} \text {. }
$$

Clearly if $G$ is not connected or has a bridge (i.e., a cut-edge) then $O D(G)$ is not finite. A celebrated theorem of Robbins [11 asserts $O D(G)$ is finite if $G$ is both connected and bridgeless. Any orientation $H$ of $G$ with $\operatorname{diam}(H)=O D(G)$ is called a minimum diameter orientation of $G$. The problem of evaluating the 
value $O D(G)$ for an arbitrary graph $G$ is very difficult. In fact it is NP-complete to determine whether a graph $G$ satisfies $O D(G) \leq 2$, [2].

Chvátal and Thomassen $[2$ initiated the investigation of the relation of the two parameters $O D(G)$ and $\operatorname{diam}(G)$. They proved that $O D(G) \leq 2(\operatorname{diam}(G))^{2}+$ $2 \operatorname{diam}(G)$ for every connected bridgeless graph $G$. Minimum diameter orientations of various families of graphs have already been studied by Gutin 6], Koh and Tan 7], Soltés 12, Plesnik [10, Boesch and Tindell [1, and others. We direct the reader to the survey of Koh and Tay [8] for a comprehensive discussion on the minimum diameter orientations of graphs.

A graph $G$ is an interval graph if there is a family of intervals $I_{v}, v \in V(G)$, in a real line such that two vertices $u, v$ are adjacent in $G$ if and only if $I_{u}, I_{v}$ overlap. If such a family of intervals can be chosen so that no interval is completely contained in another, then the graph $G$ is called a proper interval graph. Recently, Fomin et al. 3] proved that, for every connected bridgeless interval graph $G$, $O D(G) \leq \frac{3}{2} \operatorname{diam}(G)+\frac{25}{2}$ and, for every 2-connected proper interval graph $G$, $O D(G) \leq \frac{5}{4} \operatorname{diam}(G)+\frac{29}{2}$.

The purpose of this paper is to sharpen these two upper bounds. More precisely, we show that $O D(G) \leq\left\lceil\frac{3}{2} \operatorname{diam}(G)\right\rceil+1$ for every connected bridgeless interval graph $G$. There are (proper) interval graphs of arbitrarily larger diameter achieving this bound. Further, we show that, for every 2-connected proper interval graph $G, O D(G) \leq\left\lceil\frac{5}{4} \operatorname{diam}(G)\right\rceil+1$ if $\operatorname{diam}(G) \leq 3$ and $O D(G) \leq$ $\left\lceil\frac{5}{4} \operatorname{diam}(G)\right\rceil$, otherwise. Again the bounds can be attained by appropriate 2connected proper interval graphs.

\section{Connected Bridgeless Interval Graphs}

Interval graphs can be characterized in many ways, cf. [5] . According to Gilmore and Hoffman [4, a graph $G$ is an interval graph if and only if the maximal cliques of $G$ can be linearly ordered so that, for every vertex $v$ of $G$, the maximal cliques containing vertex $v$ occur consecutively in the ordering.

Let $G$ be a connected bridgeless interval graph and let $\mathcal{C}: C_{1}, C_{2}, \ldots, C_{r}$ be an ordering of the maximal cliques of $G$ that satisfies the above-mentioned property. Since $G$ is bridgeless, every $C_{i}$ has at least 3 vertices. When $r=1, G$ is a complete graph, i.e., $G=K_{n}$ where $n$ is the number of vertices of $G$. Plesnik [10] has proved that $O D\left(K_{4}\right)=3$, and $O D\left(K_{n}\right)=2$ for all $n \geq 3$ and $n \neq 4$ (cf. also [19]). A minimum diameter orientation of $K_{4}$ is depicted in Figure 1.

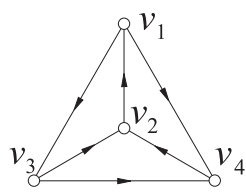

Fig. 1. $K_{4}$ and its minimum diameter orientation 
Suppose that $G$ is not a complete graph, i.e., $r \geq 2$. Let $\mathcal{C}^{\prime}: C_{i_{1}}, C_{i_{2}}, \ldots, C_{i_{d}}$ with $1=i_{1}<i_{2}<\cdots<i_{d}=r$ be chosen such that $C_{i_{j}} \cap C_{i_{j+1}} \neq \emptyset$ and $C_{i_{j}} \cap C_{i_{j+1}+1}=\emptyset$ for $1 \leq j \leq d-1$. Clearly, $d=\operatorname{diam}(G)$. Denote $S_{j}=$ $C_{i_{j}} \cap C_{i_{j+1}}, 1 \leq j \leq d-1$. Then $S_{j}$ is contained in $C_{m}$ for $i_{j} \leq m \leq i_{j+1}$. Since $C_{i_{j}} \cap C_{i_{j+1}+1}=\emptyset, S_{j} \cap S_{j+1}=\emptyset$ for $1 \leq j \leq d-1$.

When $\left|S_{j}\right|=1$, denote by $z_{j}$ the only vertex in $S_{j}$; otherwise when $\left|S_{j}\right| \geq 2$, choose arbitrarily two vertices $z_{j}, z_{j}^{\prime}$ in $S_{j}$. Call $C_{i_{j}}$ bad if $\left|S_{j-1}\right|=\left|S_{j}\right|=1$ $(2 \leq j \leq d-1)$, and good otherwise. Denote by $\ell$ the number of bad maximal cliques (in $\mathcal{C}^{\prime}$ ). Then $\ell \leq d-2=\operatorname{diam}(G)-2$.

We orient $G$ using the following steps:

(1) If $\left|S_{1}\right|=1$, arbitrarily choose a vertex $w \in C_{1}-\left\{z_{1}\right\}$ let $w \rightarrow x \rightarrow z_{1} \rightarrow w$ for all $x \in C_{1}-\left\{w, z_{1}\right\}$; if $\left|S_{1}\right| \geq 2$, let $z_{1} \rightarrow z_{1}^{\prime} \rightarrow x \rightarrow z_{1}$ for all $x \in C_{1}-\left\{z_{1}, z_{1}^{\prime}\right\}$. Note that this gives a partial orientation of $C_{1}$. Similarly, if $\left|S_{d-1}\right|=1$, arbitrarily choose a vertex $w \in C_{r}-\left\{z_{d-1}\right\}$ let $w \rightarrow x \rightarrow z_{d-1} \rightarrow w$ for all $x \in C_{r}-\left\{w, z_{d-1}\right\}$; if $\left|S_{d-1}\right| \geq 2$, let $z_{d-1} \rightarrow z_{d-1}^{\prime} \rightarrow x \rightarrow z_{d-1}$ for all $x \in C_{r}-\left\{z_{d-1}, z_{d-1}^{\prime}\right\}$. Again this gives a partial orientation of $C_{r}$.

(2) For the first $\left\lfloor\frac{\ell}{2}\right\rfloor$ bad cliques $C_{i_{j}}$, orient $v \rightarrow z_{j-1} \rightarrow z_{j} \rightarrow v$ for $v \in$ $C_{i_{j}}-\left\{z_{j-1}, z_{j}\right\}$ and for the remaining $\left\lceil\frac{\ell}{2}\right\rceil$ bad maximal cliques $C_{i_{j}}$, orient $v \rightarrow z_{j} \rightarrow z_{j-1} \rightarrow v$ for $v \in C_{i_{j}}-\left\{z_{j-1}, z_{j}\right\}$

(3) For each good clique $C_{i_{j}}$ with $\left|S_{j-1}\right|=1$ and $\left|S_{j}\right| \geq 2$, orient $v \rightarrow z_{j} \rightarrow$ $z_{j}^{\prime} \rightarrow v$ for any $v \in C_{i_{j}}-\left\{z_{j}, z_{j}^{\prime}\right\}$. For each good clique with $\left|S_{j}\right|=1$ and $\left|S_{j-1}\right| \geq 2$, orient $v \rightarrow z_{j-1} \rightarrow z_{j-1}^{\prime} \rightarrow v$ for $v \in C_{i_{j}}-\left\{z_{j-1}, z_{j-1}^{\prime}\right\}$;

(4) For each good clique $C_{i_{j}}$ with $\left|S_{j-1}\right| \geq 2$ and $\left|S_{j}\right| \geq 2$, we let $z_{j-1} \rightarrow$ $z_{j-1}^{\prime}, z_{j-1} \rightarrow z_{j} \rightarrow z_{j}^{\prime} \rightarrow z_{j-1}^{\prime}$, and $\left\{z_{j-1}^{\prime}, z_{j}^{\prime}\right\} \rightarrow v \rightarrow\left\{z_{j-1}, z_{j}\right\}$ for all $v \in C_{i_{j}}-\left\{z_{j}, z_{j}^{\prime}, z_{j-1}, z_{j-1}^{\prime}\right\}$

(5) For each $C_{m} \in \mathcal{C}-\mathcal{C}^{\prime}$, we must have $i_{j}<m<i_{j+1}$ for some $j$. (Note that the ordering of maximal cliques ensures that $C_{m}$ contains $S_{j}$ and in particular $z_{j}$ and $z_{j}^{\prime}$.) When $\left|S_{j}\right| \geq 2$, for each $v \in C_{m}-\left(C_{i_{1}} \cup C_{i_{2}} \cup \ldots \cup C_{i_{d}}\right)$, let $v \rightarrow z_{j} \rightarrow z_{j}^{\prime} \rightarrow v$. When $\left|S_{j}\right|=1$, we select a vertex $w \in C_{m}-\left\{z_{j}^{\prime}\right\}$ in such a way that the preference is given to a vertex in $C_{i_{j}} \cup C_{i_{j+1}}$. Note that the edge $w z_{j}$ may or may not be oriented by the previous steps. If it has not been oriented, then we orient it arbitrarily. Now for each vertex $v \in C_{m}-\left(C_{i_{1}} \cup C_{i_{2}} \cup \ldots \cup C_{i_{d}}\right)$, we assign directions to edges $v w, v z_{j}$ in such a way that $v, w, z_{j}$ form a directed triangle;

(6) Finally, for each edge which has not been oriented by the above steps, we assign arbitrarily a direction to it.

Lemma 1. Let $G$ be a connected bridgeless interval graph which is not complete and let $H$ be an orientation of $G$ obtained using the above steps. Then

$$
\operatorname{diam}(H) \leq\left\lceil\frac{3}{2} \operatorname{diam}(G)\right\rceil+1 .
$$

Proof. Let $v$ be a vertex of $G$. Then $v \in C_{i}$ for some $1 \leq i \leq r$. Assume $i_{j} \leq i \leq i_{j+1}$, i.e., $v \in C_{i_{j}} \cup C_{i_{j}+1} \cup \cdots \cup C_{i_{j+1}}$. When $\left|S_{j}\right|=1$, it follows from 

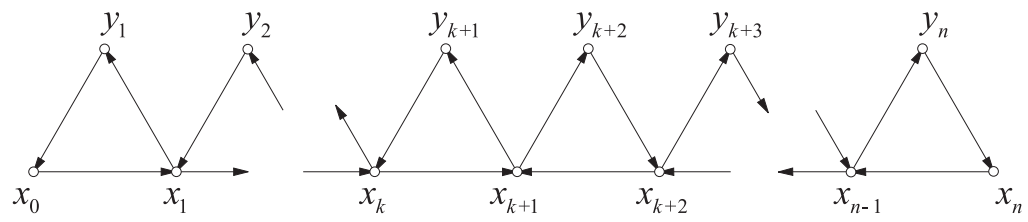

Fig. 2. A minimum diameter orientation of an interval graph $G$ of diameter $n$ showing $O D(G)=\left\lceil\frac{3}{2} n\right\rceil+1$, where $k=\left\lfloor\frac{n}{2}\right\rfloor$

steps $1,2,3$, and 5 that $d_{H}\left(v, z_{j}\right) \leq 2$ and $d_{H}\left(z_{j}, v\right) \leq 2$; when $\left|S_{j+1}\right| \geq 2$, by steps $1,3,4$, and $5, d_{H}\left(v, z_{j}\right) \leq 1, d_{H}\left(z_{j}^{\prime}, v\right) \leq 1$, and $d_{H}\left(z_{j}, z_{j}^{\prime}\right)=1$.

Let $u, v$ be any two vertices of $G$. Then $u \in C_{a}$ and $v \in C_{b}$ for some $1 \leq a, b \leq$ $r$. If $i_{j} \leq a, b \leq i_{j+1}$ for some $j=1,2, \ldots, d-1$, then

$$
d_{H}(u, v) \leq \begin{cases}d_{H}\left(u, z_{j}\right)+d_{H}\left(z_{j}, v\right) \leq 4 & \text { if }\left|S_{j}\right|=1 \\ d_{H}\left(u, z_{j}\right)+d_{H}\left(z_{j}, z_{j}^{\prime}\right)+d_{H}\left(z_{j}^{\prime}, v\right) \leq 3 & \text { if }\left|S_{j}\right| \geq 2 .\end{cases}
$$

Suppose that $i_{j} \leq a<i_{j+1} \leq i_{k} \leq b<i_{k+1}$ where $1 \leq j<k \leq d-1$. By step $2, d_{H}\left(z_{j}, z_{k}\right) \leq \operatorname{diam}(G)-2+\left\lceil\frac{\ell}{2}\right\rceil$ and $d_{H}\left(z_{k}, z_{j}\right) \leq \operatorname{diam}(G)-2+\left\lfloor\frac{\ell}{2}\right\rfloor$. Hence

$$
\begin{aligned}
d_{H}(u, v) & \leq d_{H}\left(u, z_{j}\right)+d_{H}\left(z_{j}, z_{k}\right)+d_{H}\left(z_{k}, v\right) \leq 2+\operatorname{diam}(G)-2+\left\lceil\frac{\ell}{2}\right\rceil+2 \\
& \leq 2+\operatorname{diam}(G)+\left\lceil\frac{\operatorname{diam}(G)-2}{2}\right\rceil=\left\lceil\frac{3}{2} \operatorname{diam}(G)\right\rceil+1,
\end{aligned}
$$

and

$$
\begin{aligned}
d_{H}(v, u) & \leq d_{H}\left(v, z_{k}\right)+d_{H}\left(z_{k}, z_{j}\right)+d_{H}\left(z_{j}, u\right) \leq 2+\operatorname{diam}(G)-2+\left\lfloor\frac{\ell}{2}\right\rfloor+2 \\
& \leq 2+\operatorname{diam}(G)+\left\lfloor\frac{\operatorname{diam}(G)-2}{2}\right\rfloor \leq\left\lceil\frac{3}{2} \operatorname{diam}(G)\right\rceil+1 .
\end{aligned}
$$

Therefore $\operatorname{diam}(H) \leq\left\lceil\frac{3}{2} \operatorname{diam}(G)\right\rceil+1$.

Theorem 1. Let $G$ be a connected bridgeless interval graph. Then

$$
O D(G) \leq\left\lceil\frac{3}{2} \operatorname{diam}(G)\right\rceil+1 .
$$

Proof. The statement follows from Lemma 1 and the fact that $O D\left(K_{n}\right) \leq 3$.

The sharpness of the upper bound given in Theorem 1 is shown by Figure 2, where an orientation of diameter $\left\lceil\frac{3}{2} n\right\rceil+1$ is given for an interval graph of diameter $n$.

\section{2-Connected Proper Interval Graphs}

In this section, we focus on the minimum diameter orientations of 2-connected proper interval graphs $G$. We shall adopt the same notations as in Section 2 . 
In particular, $C_{1}, C_{2}, \ldots, C_{r}$ is an ordering of the maximal cliques of $G$ in the sense of Gilmore and Hoffman. Since $G$ is a proper interval graph, the ordering is essentially unique. We also let $C_{i_{1}}, C_{i_{2}}, \ldots, C_{i_{d}}$ be the cliques as defined in Section 2. We begin with the lemma (which does not assume $G$ is 2-connected).

Lemma 2. Let $G$ be a connected proper interval graph. Then for $1 \leq j \leq d-1$ and $i_{j}<m<i_{j+1}, C_{m} \subseteq C_{i_{j}} \cup C_{i_{j+1}}$.

Proof. Suppose that $C_{m}$ contains a vertex $w$ that is not in $C_{i_{j}} \cup C_{i_{j+1}}$. Since $w$ is not in $C_{i_{j}} \cup C_{i_{j+1}}, w$ is not adjacent to a vertex $x \in C_{i_{j}}$ and not adjacent to a vertex $y \in C_{i_{j+1}}$. Let $z$ be any vertex in $C_{i_{j}} \cap C_{i_{j+1}}$. Since $C_{i_{j}} \cap C_{i_{j+1}} \subseteq C_{m}$, $z$ is in $C_{m}$ and hence adjacent to $w$. So $z$ is adjacent to $w, x$, and $y$. Since $G$ is a proper interval graph, it does not contain an induced $K_{1,3}$. This implies that $x$ and $y$ are adjacent (as otherwise the vertices $w, x, y, z$ induce a $K_{1,3}$ ). In particular, $x, y, z$ are pairwise adjacent.

Consider a maximal clique $C_{m^{\prime}}$ containing $x, y, z$. Note $C_{m^{\prime}}$ is distinct from $C_{i_{j}}, C_{m}$ and $C_{i_{j+1}}$. If $m^{\prime}>m$, then $x$ is must be in $C_{m}$ and hence adjacent to $w$, a contradiction. On the other hand, if $m^{\prime}<m$, then $y$ is in $C_{m}$ and adjacent to $w$, also a contradiction.

From now on, we assume $G$ is a 2-connected proper interval graph. Lemma 2 implies that $V(G)=C_{i_{1}} \cup C_{i_{2}} \cup \cdots \cup C_{i_{d}}$. As in Section 2, if $\left|S_{j}\right|=\left|C_{i_{j}} \cap C_{i_{j+1}}\right| \geq 2$, let $z_{j}, z_{j}^{\prime}$ be any two fixed vertices in $S_{j}$, and if $\left|S_{j}\right|=1$, let $z_{j}$ be the only vertex in $S_{j}$. Since $G$ is 2 -connected, in the case when $\left|S_{j}\right|=1$, there exist $x_{j} \in C_{i_{j}}-\left\{z_{j}\right\}$ and $x_{j+1}^{\prime} \in C_{i_{j+1}}-\left\{z_{j}\right\}$ such that $x_{j}$ and $x_{j+1}^{\prime}$ are adjacent.

We next show how to orient $G$ and to do so we consider several cases. In each case, we only give a partial orientation of $G$ and the orientations of the remaining edges may be given arbitrarily.

Case 1. $\operatorname{diam}(G)=2$.

- (1) If $\left|S_{1}\right|=1$, let $x_{2}^{\prime} \rightarrow x_{1}, v \rightarrow z_{1} \rightarrow w$ for all $v \in C_{i_{1}}-z_{1}$ and $w \in C_{i_{2}}-z_{1}, x_{1} \rightarrow v$ for all $v \in C_{i_{1}}-\left\{x_{1}, z_{1}\right\}$, and $w \rightarrow x_{2}^{\prime}$ for all $w \in C_{i_{2}}-\left\{x_{2}^{\prime}, z_{1}\right\}$.

- (2) If $\left|S_{1}\right| \geq 2$, let $v \rightarrow z_{1} \rightarrow z_{1}^{\prime} \rightarrow v$ for all $v \in V(G) \backslash\left\{z_{1}, z_{1}^{\prime}\right\}$.

Case 2. $\operatorname{diam}(G)=3$.

- (1) Suppose $\left|S_{1}\right|=\left|S_{2}\right|=1$. Note that it is possible to select $x_{2}^{\prime}, x_{2}$ from $C_{i_{2}}-\left\{z_{1}, z_{2}\right\}$. Let $x_{1} \rightarrow z_{1} \rightarrow x_{2}^{\prime} \rightarrow x_{1}, z_{1} \rightarrow z_{2}$, and $x_{2} \rightarrow x_{3}^{\prime} \rightarrow z_{2} \rightarrow x_{2}$. For all $u \in C_{i_{1}}-\left\{x_{1}, z_{1}\right\}$ let $x_{1} \rightarrow u \rightarrow z_{1}$, for all $v \in C_{i_{2}}-\left\{z_{1}, z_{2}, x_{2}^{\prime}, x_{2}\right\}$ let $z_{2} \rightarrow v \rightarrow z_{1}$, and for all $w \in C_{i_{3}}-\left\{z_{2}, x_{3}^{\prime}\right\}$ let $x_{3}^{\prime} \rightarrow w \rightarrow z_{2}$.

- (2) Suppose that exactly one of $\left|S_{1}\right|$ and $\left|S_{2}\right|$ equals 1 . Assume $\left|S_{1}\right|=1$ (the case when $\left|S_{2}\right|=1$ can be handled in a similar way. Let $v \rightarrow z_{1} \rightarrow x_{1} \rightarrow v$ for $v \in C_{i_{1}}-\left\{z_{1}, x_{1}\right\}, u \rightarrow z_{2} \rightarrow z_{2}^{\prime} \rightarrow u$ for $u \in C_{i_{2}} \cup C_{i_{3}}-\left\{z_{2}, z_{2}^{\prime}\right\}$.

- (3) Suppose $\left|S_{1}\right| \geq 2$ and $\left|S_{2}\right| \geq 2$. Let $z_{1} \rightarrow z_{2}$ and $z_{2}^{\prime} \rightarrow z_{1}^{\prime}$. Let $v \rightarrow$ $z_{1} \rightarrow z_{1}^{\prime} \rightarrow v$ for $v \in C_{i_{1}}-\left\{z_{1}, z_{1}^{\prime}\right\}$, and $u \rightarrow z_{2} \rightarrow z_{2}^{\prime} \rightarrow u$ for $u \in$ $C_{i_{2}} \cup C_{i_{3}}-S_{1}-\left\{z_{2}, z_{2}^{\prime}\right\}$. 
Case 3. $\operatorname{diam}(G) \geq 4$.

Let $C_{i_{k}}$ be a bad clique with $3 \leq k \leq d-3$. Call $C_{i_{k}}$ 2-bad if $C_{i_{k+1}}$ is also bad. Choose a set $\mathcal{L}$ of 2 -bad cliques $C_{i_{j_{1}}}, C_{i_{j_{2}}}, \ldots, C_{i_{j_{\eta}}}$ so that the following properties are satisfied:

(P1) the set contains as many 2-bad cliques as possible;

(P2) $i_{j_{k+1}} \neq i_{j_{k}+1}$;

(P3) $i_{j_{1}}+i_{j_{2}}+\cdots+i_{j_{\eta}}$ is minimum.

Note that $\mathcal{L}$ does not contain all 2-bad cliques. For instance, if $C_{i_{k-1}}$ is in $\mathcal{L}$, then $C_{i_{k}}$ cannot be in $\mathcal{L}$ even though it may be 2 -bad. Denote by $\mathcal{M}$ be the set of all bad but not 2-bad cliques $C_{i_{k}}$ with $3 \leq k \leq d-2$ such that $C_{i_{k-1}}$ is not in $\mathcal{L}$ and $|\mathcal{M}|=\ell$. Then the number of good cliques in $\mathcal{C}^{\prime}-\left\{C_{i_{1}}, C_{i_{2}}, C_{i_{d-1}}, C_{i_{d}}\right\}$ is $d-4-2 \eta-\ell$. When $C_{i_{k}} \in \mathcal{M}$, we have $C_{i_{k+1}}$ is either good or $C_{i_{d-1}}$ and hence $\ell \leq(d-4-2 \eta-\ell)+1$.

- (1) If $\left|S_{1}\right|=1$, let $x_{2}^{\prime} \rightarrow x_{1} \rightarrow z_{1}, x_{1} \rightarrow v \rightarrow z_{1}$ for all $v \in C_{i_{1}}-\left\{z_{1}, x_{1}\right\}$, and $u \rightarrow x_{2}^{\prime} \rightarrow z_{1} \rightarrow u$ for all $u \in C_{i_{2}}-\left\{z_{2}, x_{2}^{\prime}\right\}$; If $\left|S_{1}\right| \geq 2$, let $z_{1}^{\prime} \rightarrow v \rightarrow z_{1}$ for all $v \in C_{i_{1}}-\left\{z_{1}^{\prime}, z_{1}\right\}$, and $u \rightarrow z_{1}^{\prime} \rightarrow z_{1} \rightarrow u$ for all $u \in C_{i_{2}}-S_{1}$.

- (2) If $\left|S_{d-1}\right|=1$, let $x_{d-1} \rightarrow x_{d}^{\prime} \rightarrow z_{d-1}, v \rightarrow x_{d-1} \rightarrow z_{d-1} \rightarrow v$ for all $v \in C_{i_{d-1}}-\left\{z_{d-1}, x_{d-1}^{\prime}\right\}$, and $x_{d}^{\prime} \rightarrow u \rightarrow z_{d-1}$ for all $u \in C_{i_{d}}-\left\{z_{d-1}, x_{d}^{\prime}\right\}$; If $\left|S_{d-1}\right| \geq 2$, let $v \rightarrow z_{d-1}^{\prime} \rightarrow z_{d-1} \rightarrow v$ for all $v \in C_{i_{d-1}}-\left\{z_{d-1}, z_{d-1}^{\prime}\right\}$ and $z_{d-1}^{\prime} \rightarrow u \rightarrow z_{d-1}$ for all $u \in C_{i_{d}}-S_{d-1}$.

- (3) Suppose $C_{i_{j}} \in \mathcal{L}$. We only show how to give orientations of the edges in $C_{i_{j}}$ and $C_{i_{j+1}}$ for $i_{j_{1}} \leq i_{j} \leq i_{j_{\left\lceil\frac{\eta}{2}\right\rceil}}$. (When $i_{j_{\left\lceil\frac{\eta}{2}\right\rceil}}<i_{j} \leq i_{j_{\eta}}$, the edges are oriented reversely.) Let $x_{i_{j+1}}^{\prime} \rightarrow x_{i_{j}}, v \rightarrow z_{j-1} \rightarrow z_{j} \rightarrow v$ for all $v \in$ $C_{i_{j}}-\left\{z_{j}, z_{j-1}\right\}$, and $u \rightarrow z_{j} \rightarrow z_{j+1} \rightarrow u$ for all $u \in C_{i_{j+1}}-\left\{z_{j}, z_{j+1}\right\}$.

- (4) For the first (with respect to the order of $\mathcal{C}$ ) $\left\lfloor\frac{\ell}{2}\right\rfloor$ cliques $C_{i_{k}}$ in $\mathcal{M}$, let $v \rightarrow z_{k-1} \rightarrow z_{k} \rightarrow v$ for all $v \in C_{i_{k}}-\left\{z_{k-1}, z_{k}\right\}$; for the other cliques in $\mathcal{M}$, we orient the edges reversely.

- (5) Suppose that $C_{i_{j}}$ with $3 \leq j \leq d-2$ is a good clique. If $\left|S_{j-1}\right|=1$ and $\left|S_{j}\right| \geq 2$, let $v \rightarrow z_{j} \rightarrow z_{j}^{\prime} \rightarrow v$ for any $v \in C_{i_{j}}-\left\{z_{j}, z_{j}^{\prime}\right\}$; if $\left|S_{j}\right|=1$ and $\left|S_{j-1}\right| \geq 2$, let $v \rightarrow z_{j-1} \rightarrow z_{j-1}^{\prime} \rightarrow v$ for $v \in C_{i_{j}}-\left\{z_{j-1}, z_{j-1}^{\prime}\right\}$; if $\left|S_{j-1}\right| \geq 2$ and $\left|S_{j}\right| \geq 2$, let $z_{j-1} \rightarrow z_{j-1}^{\prime}, z_{j-1} \rightarrow z_{j} \rightarrow z_{j}^{\prime} \rightarrow z_{j-1}^{\prime}$, and $\left\{z_{j-1}^{\prime}, z_{j}^{\prime}\right\} \rightarrow v \rightarrow\left\{z_{j-1}, z_{j}\right\}$ for all $v \in C_{i_{j}}-\left\{z_{j}, z_{j}^{\prime}, z_{j-1}, z_{j-1}^{\prime}\right\}$.

Theorem 2. Let $G$ be a 2-connected proper interval graph. Then

$$
O D(G) \leq \begin{cases}\left\lceil\frac{5}{4} \operatorname{diam}(G)\right\rceil+1 & \text { if } \operatorname{diam}(G) \leq 3 \\ \left\lceil\frac{5}{4} \operatorname{diam}(G)\right\rceil & \text { if } \operatorname{diam}(G) \geq 4\end{cases}
$$

Proof. When $G$ is complete, $O D(G) \leq 3 \leq\left\lceil\frac{5}{4} \operatorname{diam}(G)\right\rceil+1$ by Theorem 1 . When $G$ is not complete and satisfies $\operatorname{diam}(G) \leq 3$, it can be verified easily from the orientations given in Case 1 and Case 2 that $O D(G) \leq\left\lceil\frac{5}{4} \operatorname{diam}(G)\right\rceil+1$. So suppose that $\operatorname{diam}(G) \geq 4$.

Let $u, v$ be any two vertices of $G$. Without loss of generality assume $u \in C_{i_{j}}$ and $v \in C_{i_{k}}$ for some $j \leq k$. When $k-1 \leq j \leq k$, according to steps 1 and 2 in Case $3, d_{H}(u, v) \leq 5$ if $j=k=1$ or $j=k=d$ and, according steps 3,4 , and 5 in Case $3, d_{H}(u, v) \leq 3$ if $j=k \neq 1, d$. When $j=k-1, d_{H}(u, v) \leq 4$ according to steps $1-5$ in Case 3. 
Suppose now $j<k-1$. Let $u^{\prime} \in C_{i_{1}}-\left\{z_{1}, x_{1}\right\}$ if $\left|S_{1}\right|=1$ and $u^{\prime} \in C_{i_{1}}-S_{1}$ if $\left|S_{1}\right| \geq 2$, and let $v^{\prime} \in C_{i_{d}}-\left\{z_{d-1}, x_{d}^{\prime}\right\}$ if $\left|S_{d-1}\right|=1$ and $v^{\prime} \in C_{i_{d}}-S_{d-1}$ if $\left|S_{d-1}\right| \geq 2$.

Claim: $d_{H}(u, v) \leq d_{H}\left(u^{\prime}, v^{\prime}\right)$ and $d_{H}(v, u) \leq d_{H}\left(v^{\prime}, u^{\prime}\right)$.

Proof of Claim: We only prove $d_{H}(u, v) \leq d_{H}\left(u^{\prime}, v^{\prime}\right)$ as the proof of the other inequality is similar. Denote $P_{t}^{\prime \prime}=C_{i_{t}} \cup C_{i_{t+1}}$ with $C_{i_{t}} \in \mathcal{L}$. If $u$ does not lie in $P_{t}^{\prime \prime}$ for any $t$, then $d_{H}\left(u, z_{j}\right) \leq 2 \leq d_{H}\left(u^{\prime}, z_{j}\right)$; If $u$ lies in $P_{t}^{\prime \prime}$ for some $t$, then $3 \leq t \leq d-3$ and either $t=j$ or $t=j-1$, so $d_{H}\left(u, z_{t+1}\right) \leq 3 \leq d_{H}\left(u^{\prime}, z_{t+1}\right)$.

If $v$ does not lie in $P_{t}^{\prime \prime}$ for any $t$, then $d_{H}\left(z_{k-1}, v\right) \leq 2 \leq d_{H}\left(z_{k-1}, v^{\prime}\right)$; If $v$ lies in $P_{p}^{\prime \prime}$ for some $p$, then $3 \leq p \leq d-3$ and either $p=k$ or $p=k-1$, so $d_{H}\left(z_{p-1}, v\right) \leq 3 \leq d_{H}\left(z_{p-1}, v^{\prime}\right)$. So

$$
\begin{aligned}
d_{H}(u, v) & \leq d_{H}\left(u, z_{r}\right)+d_{H}\left(z_{r}, z_{m}\right)+d_{H}\left(z_{m}, v\right) \\
& \leq d_{H}\left(u^{\prime}, z_{r}\right)+d_{H}\left(z_{r}, z_{m}\right)+d_{H}\left(z_{m}, v^{\prime}\right)=d_{H}\left(u^{\prime}, v^{\prime}\right)
\end{aligned}
$$

where $r=t+1$ if $u$ lies in $P_{t}^{\prime \prime}$ for some $t$, and $r=j$ otherwise; $m=p-1$ if $v$ lies in $P_{p}^{\prime \prime}$ for some $p$, and $m=k-1$ otherwise.

To estimate $d_{H}\left(u^{\prime}, v^{\prime}\right)$ and $d_{H}\left(v^{\prime}, u^{\prime}\right)$, first we have

$$
\begin{aligned}
d_{H}\left(u^{\prime}, v^{\prime}\right) & =d_{H}\left(u^{\prime}, z_{2}\right)+d_{H}\left(z_{2}, z_{d-2}\right)+d_{H}\left(z_{d-2}, v^{\prime}\right) \\
& \leq 2+2\left\lfloor\frac{\eta}{2}\right\rfloor+3\left\lceil\frac{\eta}{2}\right\rceil+\left\lceil\frac{\ell}{2}\right\rceil+2\left\lfloor\frac{\ell}{2}\right\rfloor+(d-4-2 \eta-\ell)+3 \\
& =1+\left\lceil\frac{\eta}{2}\right\rceil+\left\lfloor\frac{\ell}{2}\right\rfloor+d .
\end{aligned}
$$

Since $\ell \leq(d-4-2 \eta-\ell)+1$, we have

$d_{H}\left(u^{\prime}, v^{\prime}\right) \leq 1+\left\lceil\frac{\eta}{2}\right\rceil+\left\lfloor\frac{d-3-2 \eta}{4}\right\rfloor+d \leq 1+\left\lceil\frac{\eta}{2}\right\rceil+\left\lceil\frac{d}{4}\right\rceil-\left\lceil\frac{3}{4}+\frac{\eta}{2}\right\rceil+d=\left\lceil\frac{5}{4} \operatorname{diam}(G)\right\rceil$.

Similarly, $d_{H}\left(v^{\prime}, u^{\prime}\right) \leq 1+\left\lfloor\frac{\eta}{2}\right\rfloor+\left\lceil\frac{\ell}{2}\right\rceil+d$ and hence

$d_{H}\left(v^{\prime}, u^{\prime}\right) \leq 1+\left\lceil\frac{\ell}{2}\right\rceil+\left\lfloor\frac{d-3-2 \ell}{4}\right\rfloor+d \leq 1+\left\lceil\frac{\ell}{2}\right\rceil+\left\lceil\frac{d}{4}\right\rceil-\left\lceil\frac{3}{4}+\frac{\ell}{2}\right\rceil+d=\left\lceil\frac{5}{4} \operatorname{diam}(G)\right\rceil$.

When $\operatorname{diam}(G)=1$, the upper bound of $O D(G)$ in Theorem 2 is achieved by a minimum diameter orientation of $K_{4}$ (see Figure 1). The following proposition from [3] shows that the upper bound in Theorem 2 is $\operatorname{sharp}$ for $\operatorname{diam}(G) \geq 4$.

Proposition 1. 3] For $d \geq 3$, there is a 2-connected proper interval graph $G$ with $\operatorname{diam}(G) \geq d$ and $O D(G) \geq \frac{5}{4} \operatorname{diam}(G)$.

The sharpness of upper bounds of $O D(G)$ for the cases when $2 \leq \operatorname{diam}(G) \leq 3$ is given by Propositions 2 and 3 below. Indeed, it can be verified that the digraphs in Figures 3 and 4 are the minimum diameter orientations of their underlying graphs. Therefore we have 


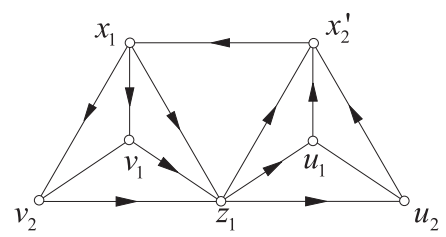

Fig. 3. A minimum diameter orientation of a 2-connected proper interval graph of diameter two, where unoriented edges may be oriented arbitrarily

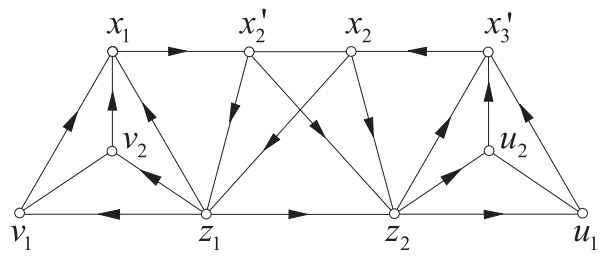

Fig. 4. A minimum diameter orientation of 2-connected proper interval graph of diameter three, where unoriented edges may be oriented arbitrarily

Proposition 2. Let $G_{1}$ be the underlying graph of the digraph in Figure 3. Then $O D\left(G_{1}\right) \geq 4$.

Proposition 3. Let $G_{2}$ be the underlying graph of the digraph in Figure 4 . Then $O D\left(G_{2}\right) \geq 5$.

\section{References}

1. F. Boesch and R. Tindell, Robbins' theorem for mixed multigraphs, Amer. Math. Monthy 87 (1980) 716-719.

2. V. Chvátal and C. Thomassen, Distances in orientations of graphs, J. Combin. Theory Ser. B 24 (1978) 61-75.

3. F.V. Fomin, M. Matamala, E. Prisner and I. Rapaport, AT-free graphs: linear bounds for the oriented diameter, Discrete Appl. Math. 141 (2004) 135-148.

4. P.C. Gilmore and A.J. Hoffman, A characterization of comparability graphs and of interval graphs, Canadian J. Math. 16(3) (1964) 539-548.

5. M.C. Golumbic, Algorithmic Graph Theory and Perfect Graphs, Academic Press, New York, 1980.

6. G. Gutin, Minimizing and maximizing the diameter in orientations of graphs, Graphs Combin. 10 (1994) 225-230.

7. K.M. Hoh and B.P. Tan, The minimum diameter of orientations of complete multipartite graphs, Graphs Combin. 12 (1996) 333-339.

8. K.M. Koh and E.G. Tay, Optimal orientations of graphs and digraphs: a survey, Graphs Combin. 18 (2002) 745-756.

9. S.B. Maurer, The king chicken theorems, Math. Mag. 53 (1980) 67 - 80. 
10. J. Plesnik, Diametrically critical tournaments, Pest. Matem. 100 (1975) 361 - 370.

11. H. E. Robbins, Theorem on graphs with an application to a problem of traffic control, Amer. Math. Monthy 46(5) (1929) 281-283.

12. L. Sotles, Orientations of graphs minimizing the radius or the diameter, Math. Slovaca 38(3) (1986) 289-296. 